\title{
Variations in Schumann resonances and their relation to atmospheric electric parameters at Nagycenk station
}

\author{
F. Märcz, G. Sátori, B. Zieger \\ Geodetic and Geophysical Research Institute of the Hungarian Academy of Sciences, H 9401 Sopron, POB 5, Hungary
}

Received: 22 May 1996 / Revised: 4 June 1997 / Accepted: 6 June 1997

\begin{abstract}
The present study is based on simultaneous measurements of the atmospheric electric potential gradient (PG) and Schumann resonances at Nagycenk station (Hungary) from 1993 to 1996. Annual and semiannual variations detected previously in the relative amplitudes of Schumann resonances (SR) in the first three modes are confirmed by the extended data series applied here. The regular annual variation found in the PG (with winter maximum and summer minimum) is in the opposite phase, compared to that of the SR amplitudes. Nevertheless, even the PG (being a parameter of the DC global circuit) occasionally shows a distinct secondary peak in summer as indicated by the results of the present analysis (and corresponding to a recent study on further parameters of the DC global circuit). In spite of the presumed dominance of local influence over the global one, a suitable PG parameter correlates well with SR (representing the AC global circuit) on the annual time scale. It also became evident that a semiannual variation (with spring and autumn maxima and winter and summer minima) is generally present in SR. Certain signatures of a semiannual variation have also been revealed in the PG, however, the phase of this semiannual variation does not fit the pattern shown by SR (and tropical surface air temperature, respectively). The representativeness of the PG data has also been checked by means of a single day's diurnal variations displaying a phase corresponding to that of the 'Carnegie Curve'. Additionally, the coincidence of short-term changes (lasting some hours) both in the $\mathrm{SR}$ and the PG parameter is also demonstrated on a day disturbed by local factors. The results are discussed in the context of correlations between surface air temperature and parameters of the atmospheric electric global circuit shown by previous studies.
\end{abstract}

\section{Introduction}

Atmospheric electric phenomena take place in a global spherical capacitor consisting of two conductors and the insulating air between them. One of the conductors is the Earth's surface and the other is represented by the highly conductive layers of the upper atmosphere. The lightning associated with thunderstorm activity generates electromagnetic waves which propagate in this spherical capacitor i.e. in the Earth - ionosphere cavity. The cavity is a wave guide for electromagnetic waves in the ELF-band $(3 \mathrm{~Hz}-30 \mathrm{kHz})$. Waves of certain frequencies become accentuated in this cavity and they are referred to as Schumann resonances. The first (basic) mode of the Schumann resonances (SR) appears as a standing wave in the Earth - ionosphere wave guide, its wavelength is commensurable with the circumference of the Earth. The resonant frequency of each mode shows changes of some tenths of $\mathrm{Hz}$ and the amplitude of the signal also changes. The frequency slip is partly due to the changes in the vertical extension of the wave guide and in the conductivity of its upper wall (i.e. in the height of the corresponding ionospheric layer and its conductivity). The frequency slip also depends on the site of the source generating the ELF waves (i.e. on the distance of thunderstorm activity from the station of observations). The latter circumstance is also influential in the amplitude of the signal, however, sometimes the amplitude is also influenced by local (atmospheric electric and meteorological) conditions.

Atmospheric electric measurements have been carried out at the Nagycenk observatory (geographical lat.: $47^{\circ} 38^{\prime}$; long.: $16^{\circ} 43^{\prime}$; Hungary) for more than three decades. The potential gradient (PG) is measured at a height of $1 \mathrm{~m}$ above the ground. (The equipment and the conditions for the observations have been described in detail in two earlier papers: Bencze and Märcz, 1967, 1981; here the most important characteristics and circumstances are listed again.) The PG equipment is 
constructed on the basis of a scheme by Koenigsfeld and Piraux (1951). Its sensor includes a radioactive collector; the response time of the sensor to electric field variations is about $20 \mathrm{~s}$. The measuring range is limited between $+300 \mathrm{~V} / \mathrm{m}$ and $-300 \mathrm{~V} / \mathrm{m}$ (positive values correspond to the fair-weather polarity, i.e. electric field is downward directed). The base line (corresponding to zero potential) is checked each day and the equipment is calibrated each week. Due to the careful and continuous service of the equipment, both the base line and the calibration values remained stable for the interval of more than three decades, thus the high quality of data could be preserved. The PG data are derived from analogue records; the data processing yields hourly mean values of the PG (in $\mathrm{V} / \mathrm{m}$ ) which are included (together with geomagnetic, Earth current and ionospheric absorption data) in the Geophysical Observatory Reports published periodically by the Geodetic and Geophysical Research Institute.

The observatory is situated in a wooded area, far from large settlements, thus influences of industrial or anthropogenic origin hardly disturb the atmospheric electricity measurements. Additionally, the area is protected by nature conservation as a National Park. This is mainly due to the neighbouring Lake Fertö which is a steppe lake, and a rather unique site in this part of Europe. The PG sensor itself is situated in a grassy, flat area, the distance between the sensor and the atmospheric electricity building (with a height of $4 \mathrm{~m}$ ) is $25 \mathrm{~m}$. Woods surrounding the observatory are sufficiently distant from the sensor (the nearest woods are about $50 \mathrm{~m}$ away), however, a small group of trees have grown up eastwards of the sensor at a distance of about $20 \mathrm{~m}$. (A long-term decline appearing in PG is most likely attributable to the latter situation, however, a secular decrease of the ionospheric potential reported by Williams and Renno, 1991, might also contribute to the PG decline. This topic will be discussed in another study.

Preliminary measurements of the Schumann resonances were carried out at the same site (in an appropriate distance from the atmospheric electric measurements) in the 1960s (Ádám and Bencze, 1963). Continuous digital recording of the first three SR modes of the vertical electric field component was started at the Nagycenk observatory in the early 1990s (Sátori et al., 1996). The measuring equipment consists of the following units: stable ball-antenna $2 \mathrm{~m}$ above the ground in a plain area, preamplifier with high input impedance and low noise, amplifier and personal computer with high speed multi-channel AD-converter. (The amplified signal in the range $2-25 \mathrm{~Hz}$ is sampled with a rate of $\mathrm{d}=10 \mathrm{~ms}$ for $35.8 \mathrm{~s}$, or until the signal surpasses the dynamic range of the $\mathrm{AD}$ converter; in the latter case the sampling is interrupted and then restarted.) The complex demodulation used as spectral filtering technique allows the determination of the time variations of the actual SR peak frequencies with great accuracy and those of the amplitudes belonging to them. Initial results have confirmed that this technique is suitable for monitoring changes and events in the first three SR modes (Sátori et al., 1996; Sátori, 1996).
Based on time series covering a period somewhat longer than a decade, variations of different periods were investigated in the Nagycenk PG record (Märcz and Bencze, 1981). Thus, a diurnal variation similar to that known from Israël (1973) as a single-oscillation diurnal variation was shown for all seasons when separating the data according to winter, equinox and summer months. Additionally, a seasonal variation with a maximum in late winter and a minimum in early summer was also detected. The presence of a semiannual variation could not be confirmed on the basis of these earlier results. In the present study, a PG data set from 1993 to 1996 is analysed. This set covered the four year interval when simultaneous SR measurements were also made at the Nagycenk observatory, thus the data of an appropriate SR parameter could also be included into the investigations.

The analysis is mainly aimed at the detection of similar signatures in the seasonal variation of the PG and the relative amplitudes of the vertical electric component of the Schumann resonances. Namely, Sátori and Zieger (1996) using an appropriate filtering technique have found an annual component in the first three modes of the latter parameter showing a maximum in Northern Hemisphere summer and a minimum in winter in accordance with the annual variation of global thunderstorm activity in the Northern Hemisphere. (Their initial analysis covered a shorter interval from May 1993 to May 1995.) Based on results published in literature (e.g. Israël, 1973) and on our earlier investigations (Märcz and Bencze, 1981), it is evident that the phase of the annual variation of the PG measured at land stations is opposite to that found in the Nagycenk SR parameter (and global thunderstorm activity) because of the dominance of local influence in the PG. The role of local air conductivity is particularly dominant in PG variations at land stations. The annual variation of air conductivity displays a winter minimum and a summer maximum as shown for a Northern Hemisphere station (at Kew) e.g. in Fig. 35 by Chalmers (1957). Unfortunately, air conductivity measurements were not carried out at Nagycenk station. However, it can be assumed that for conductivity the phase of the annual variation (at Northern Hemisphere stations) is generally like that found at Kew. Thus, the annual variation of the Nagycenk PG (with winter maximum and summer minimum) can mainly be attributed to the inverse seasonal variation of local air conductivity.

On the basis of earlier observations, Adlerman and Williams (1996) have recently shown the seasonal variation of air conductivity together with the inverse variation of Aitken nuclei during the year. In the Northern Hemisphere, the number of Aitken nuclei is generally larger in winter than in summer. Consequently, air conductivity is reduced (PG is increased) in winter while it is increased ( $\mathrm{PG}$ decreased) in summer at Northern Hemisphere stations. A reversal in the behaviour of these atmospheric electric parameters has also been shown for the Southern Hemisphere. In addition, Adlerman and Williams (1996) reanalysed the entire potential gradient data set of the Carnegie and Maud 
measurements. Since most of these measurements were carried out over the open ocean, where the aerosol variability is small, it could be supposed that the data might virtually be uncontaminated by effects of local pollution. Adlerman and Williams (1996) hoped to find a summertime maximum in these potential gradient data, as they detected a summer peak of air-Earth current in unpublished Mauna Loa data, too. In fact they confirmed that the mean potential gradient peaks during Northern Hemisphere summer rather than in winter. In the present study, we try to find a signature of this summer peak in the Nagycenk PG data, even though we are aware of the dominant influence of local factors on PG measured at a land station. This presumed summer peak would also coincide with the summer maximum shown for the AC component of the global circuit (represented by the SR parameter) in the work of Sátori and Zieger (1996). It should be mentioned that results of Adlerman and Williams (1996) revealing a summer peak in parameters of the global DC circuit show correspondence with results (Williams, 1994) indicating a strong maximum in the surface air temperature $\left( \pm 60^{\circ}\right.$ latitude) and a diffuse maximum in thunder day data $\left( \pm 60^{\circ}\right.$ latitude).

Since the PG can be regarded as a parameter directly connected with the zeroth order SR mode (DC component) of the Earth - ionosphere wave guide, it is reasonable to presume certain similarities between periodicities in the $\mathrm{PG}$ and Schumann resonances (identified as the AC component). Nevertheless, it was previously mentioned that the PG measurements carried out at land stations are affected by local influences, thus the global signal content in the DC component (as represented by $\mathrm{PG}$ data) is presumedly diminished to a greater extent in comparison with that in the $\mathrm{AC}$ component (Schumann resonances). Additionally, the true source for these two components of the global circuit may be different; consequently the PG will not always follow a one-to-one correspondence with SR. Based on both normal mode theory for the lossy cavity (Wait, 1960) and practical experience, it is also suggested that the SR signal rather depends on where it is observed relative to the sources, while in the case of PG the source positions should be less influential. The present study intends to check whether the semiannual variation (certainly present in the SR parameter) might occasionally appear in the PG, too. The analysis made by Sátori and Zieger (1996) has, indeed, revealed a semiannual signal in the relative amplitudes of the vertical electric component of SR (which was especially significant in the first and second mode). They found secondary maxima around spring and autumn, in addition to the annual maximum appearing in summer. Adlerman and Williams (1996) showed only weak evidence for a semiannual signal in the Carnegie potential gradient data with small maxima around September to October and February to March.

The diurnal variation of the PG will also be demonstrated on selected individual days in order to check the representativeness of the measurements on this basis. Namely, a PG diurnal variation similar (in phase and amplitude) to the unitary diurnal variation of the global circuit (as represented by the 'Carnegie Curve') could confirm that the global signal content in the Nagycenk PG data is acceptable even on this time scale. There are short-term changes in the PG which originate from local effects, especially from thunderstorm activity in the vicinity of the observation site and from meteorological disturbances. These changing local conditions might also influence certain parameters of the Schumann resonances. A search for related cases is another goal of the present study using data of the Nagycenk observatory.

\section{Data selection and processing}

In the case of atmospheric electric data, several selection methods have been introduced and applied in order to yield a suitable basis for the investigation of different atmospheric electric phenomena. Actually, certain restrictions are mostly applied which should allow only the processing of data obtained under optimum measuring conditions; consequently a part of the data is generally discarded from the measured series. The exclusion of data is mainly aimed at the selection of undisturbed or fair-weather periods when the typical properties of the corresponding atmospheric electric parameter might be most pronounced. As mentioned by Israël (1973), generally valid guide-lines have not yet been established for data selection, however, certain recommendations exist which lay emphasis on local meteorological conditions, too. Thus for investigating the typical characteristics of an atmospheric electric parameter, it is advisable to retain only data from periods without precipitation, fog, blown snow and blown dust, and with wind speeds below 3 Beaufort, cloudiness less than $3 / 10$ and not concentrated in the zenith (e.g. Dolezalek, 1991). In spite of the attempt to remove the strongest meteorological influences from the data, additional local peculiarities will be preserved in them. This means the detection of purely global effects in an atmospheric electric parameter is certainly a difficult task. The applied method of data selection also influences the comparability of results derived from data series of different stations.

Taking into account the previous considerations, it would be useful to carry out continuous meteorological observations at each atmospheric electricity station. At Nagycenk, meteorological observations were accomplished three times every day by the Hungarian Meteorological Service in the early 1960s, however, they ceased after some years. Consequently, a selection of PG data according to meteorological factors is not possible in the case of data applied in the present study, and it is even impossible when using a data set over a long interval. Nevertheless, a certain selection of data is reasonable and this has been done regularly.

In the processing of PG values measured at Nagycenk, hourly averages are determined and these are included in the periodically issued 'Observatory Reports'. The PG values are taken only from hours with a 
recording period of more than 30 minutes or more. No averages are determined for hours in which the PG exceeded the limits of $\pm 300 \mathrm{~V} / \mathrm{m}$. Such cases generally occur during local thunderstorms and during stormy rain and snow, thus hours influenced by these meteorological factors are not included in the original data base of the Nagycenk observatory. Additionally for the present study, all hourly averages have been removed from the original data base if the PG was negative (reversed to normal fair-weather direction), i.e. certainly disturbed for some reason. Since the PG usually reverses its direction during light rain (Israël, 1973), as well as PG values in hours with stormy rain or snow were in any case excluded from the original data base, it is most probable that $\mathrm{PG}$ data retained in this selected set might be effectively free from the effect of rain. Some of the disturbed (and as a consequence discarded) PG values might not directly be associated with rain; instead they were perturbed by electrified clouds appearing over the measuring area. Thus, the strongest meteorological effects are removed from the selected data, however, they are somewhat contaminated by influences of certain other meteorological factors.

On average, $80 \%$ of the possible maximum number of data are retained in the selected set, the other $20 \%$ is excluded due to these selection procedures and because of gaps in the observations. Based on this PG data set, monthly means have been determined for each hour of the day. These values will be arranged suitably for investigating the appearance of an annual and a semiannual signal in a DC parameter of the global circuit during the interval from 1993 to 1996. (Simultaneous SR data were available from May 1993.) Monthly means of the relative amplitudes of the vertical electric component of SR (in the first three modes) will be used for showing variations on the same time scale for a parameter representing the AC component of the global circuit. The data set of both components seems to be sufficient for appropriate comparisons.

\section{Results}

\subsection{Annual and semiannual variations in Schumann resonances and in the $P G$}

The monthly means of the squared relative amplitudes of the vertical electric component of SR have been determined for the interval from May 1993 to December 1996. They show clearly the presence of an annual variation with a maximum in Northern Hemisphere summer and a minimum in winter (Fig. 1). The semiannual signal, confirmed in SR by a previous analysis (Sátori and Zieger, 1996), is generally present in this longer data series, too. Maxima appearing around spring and autumn are most distinct in each mode in 1994. In the following years, the appearance of spring and autumn maxima is somewhat different in the individual modes. It seems that the amplitude of the semiannual signal decreases towards 1996. In spite of these modifications, both the annual and the semiannual

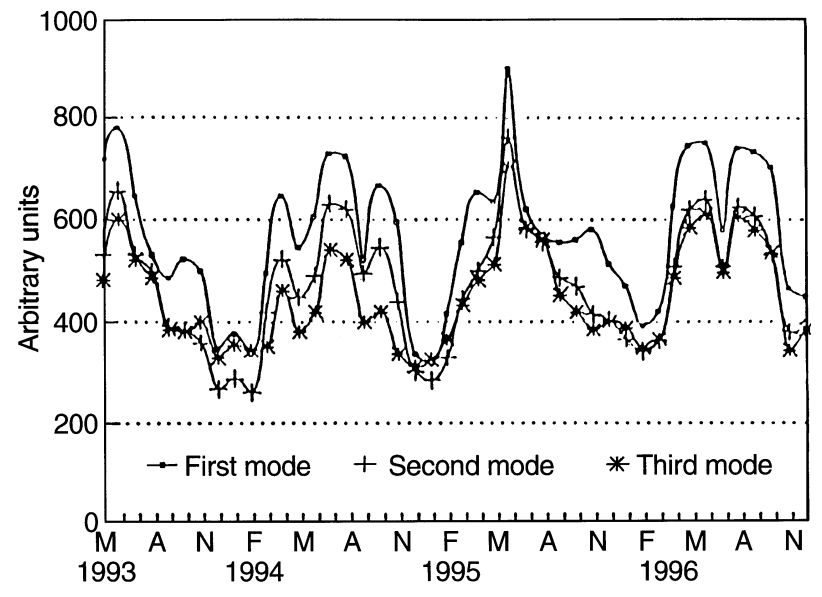

Fig. 1. Monthly means of squared relative amplitudes of the SR vertical electric component (in arbitrary units) showing the seasonal variation in the first three modes at Nagycenk station from May 1993 to December 1996

signal, revealed by an analysis using a suitable filtering technique in the work of Sátori and Zieger (1996), are preserved during the interval investigated here.

Using the selected data set of the Nagycenk PG (described in the previous section), Fig. 2 shows the monthly means of this parameter determined for each hour of the day during the interval from January 1993 to December 1996. In accordance with earlier results (Märcz and Bencze, 1981), a distinct annual variation is displayed by the envelopes of the presented curves with a maximum in Northern Hemisphere winter and a minimum in summer. Thus, the phase of the annual variation of the $\mathrm{PG}$ is opposite to that of the SR parameter in Fig. 1, and corresponding to that shown by Adlerman and Williams (1996) for PG measured at several Northern Hemisphere stations. As mentioned in the introduction, this behaviour of the $\mathrm{PG}$ can be attributed to the inverse seasonal variation of local air

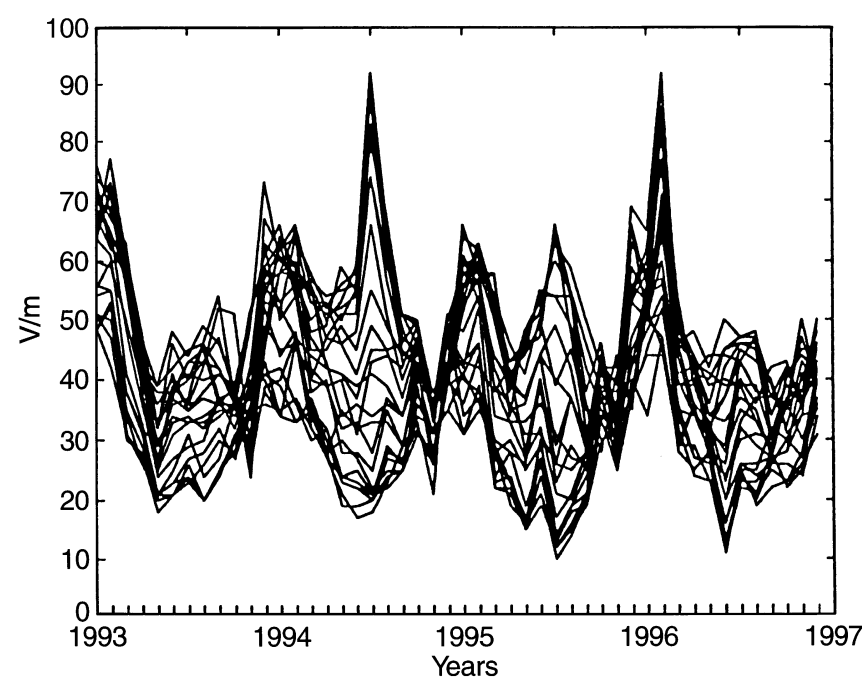

Fig. 2. Monthly means of the Nagycenk PG (in V/m) for each hour of the day in the interval between January 1993 and December 1996 
conductivity at the Northern Hemisphere (e.g. Chalmers, 1957) or to that of condensation nuclei varying in phase with the PG during the year (Adlerman and Williams, 1996). In Fig. 2, it is striking that the upper envelope of the curves also reveals peaks in summer which are especially distinct in 1994 and 1995. These peaks are reminiscent of the results of Adlerman and Williams (1996) who reanalysed the entire Carnegie and Maud potential gradient data and showed that this parameter is peak in summer rather than in winter, like the air-Earth current at the Mauna Loa station. These results are consistent with the peak of the global thunderstorm activity in the same season. Consequently, they hint at the fact that, in spite of the dominance of local influence, the global signal content of the selected Nagycenk PG data might be preserved, at least to a certain extent.

The monthly means of the PG determined for each hour of the day made it possible to plot the seasonal variation of PG separately on the basis of day-time data and night-time data, respectively. This distinction between day and night seemed to be reasonable because of the characteristic diurnal (UT) variation of global thunderstorm activity which generally shows maximum in the afternoon hours and minimum in the night or dawn hours. Figure 3 shows the seasonal variation based on PG means of day-time hours (using a $6 \mathrm{~h}$ interval between 1000 UT and 1600 UT) and on those of night hours (between 2200 UT and 0400 UT). In addition, daily means (based on $24 \mathrm{~h}$ ) are also given for each month. The curve for the latter means (solid line in Fig. 3) shows the annual variation with winter maxima and summer minima, however, secondary maxima also appear in summer, especially in 1994, 1995 and slightly in 1996. These summer peaks are most significant in the case of day-time data (dashed line), while they are missing in night hours (dotted line). To explain these results the following considerations should be considered.

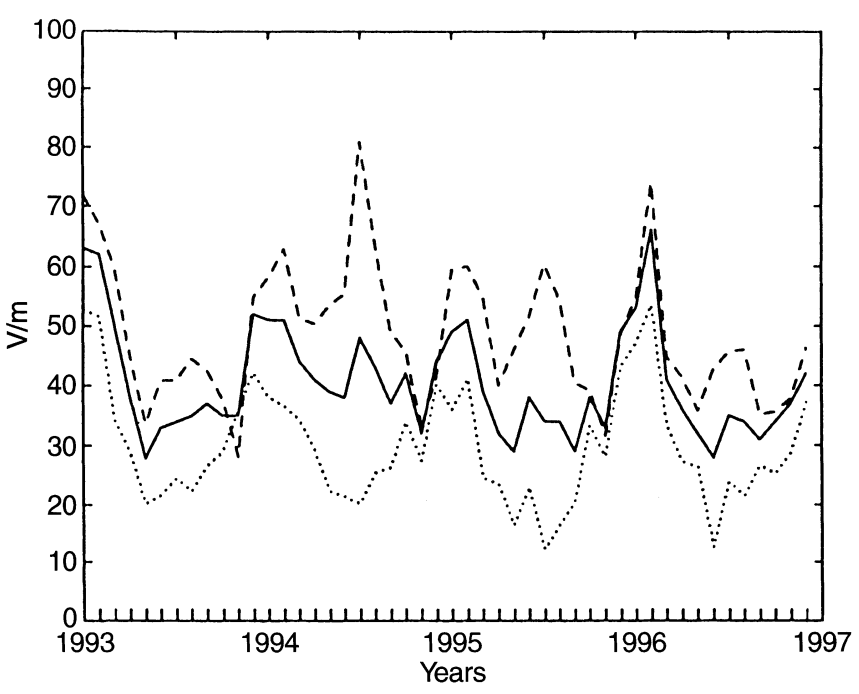

Fig. 3. Monthly means of the $\mathrm{PG}$ (in $\mathrm{V} / \mathrm{m}$ ) based on different data sets for the same interval as in Fig. 2; middle: all hours (00-24 UT); top: day-time hours (10-16 UT); bottom: night-time hours: (22-04 UT)
The seasonal variation of PG based on night data is consistent with the suggested inverse seasonal variation of local air conductivity as discussed previously, (and it is in-phase with that of condensation nuclei). In spite of the fact that high local air conductivity (and low number of condensation nuclei) in the Northern Hemisphere summer should result in decreased PG values, rather clear PG peaks appear in each summer when day-time data are used for demonstrating the seasonal variation of PG. In 1994, the summer maximum is higher than any winter maximum during the investigated interval, and in 1995 it is commensurable with some of the winter maxima. It might be supposed that these summer peaks originate from local thunderstorm activity generally occurring around and after the noon hours at Nagycenk station; however, this supposition does not hold since data contaminated by local thunderstorm effects have mostly been excluded from the original data base due to the selection procedure described in the previous section. Thus, it seems likely that the summer peaks revealed in the day-time PG are signatures associated rather with global thunderstorm activity. This resembles similar results shown by Adlerman and Williams (1996) on the basis of Carnegie and Maud PG data. It should be mentioned that the day-time interval (used for showing the seasonal behaviour of the Nagycenk PG between 1000 UT and 1600 UT) covers a period when thunderstorms can be expected not only on the European but also on the African continent, as shown e.g. in Fig. 158 of Israël (1973). It is most likely that the enhanced African contribution to global thunderstorm activity is involved in the generation of occasional summer peaks in the day-time Nagycenk PG.

SR intensity, as well as the global thunderstorm activity has a night-time or early dawn minimum (in UT) and a maximum in the afternoon hours. It can be assumed with reason that a global contribution can be significant, in addition to local effects, in the seasonal variation of day-time PG, while it is quite limited in the seasonal variation of night-time PG. This is supported by the fact that the anomalous summer maxima attributed to global (or at least regional) effects occur only in the day-time PG series and not in the night-time PG data, as shown in Figs. 2 and 3. Thus, provided that local effects in PG have similar seasonal variations during day-time and night-time, the local effects can be minimised in the seasonal variation of PG by computing the normalised difference between day-time and nighttime monthly PG data. Due to the minimised local contribution, the parameter obtained can be regarded as indicator of the global signal in the seasonal variation of PG. As shown in Fig. 4, this parameter is well correlated with the average SR amplitude computed from the first three SR modes, confirming the connection between the DC and AC components of the global circuit on an annual time scale. The corresponding cross-correlation coefficient is 0.44 ; based on a Student's test, it is significant at a confidence level of 99 per cent.

As seen in Fig. 1, a semiannual signal is indicated by spring and autumn maxima in the SR parameter. Inspecting thoroughly the changes of the monthly PG 


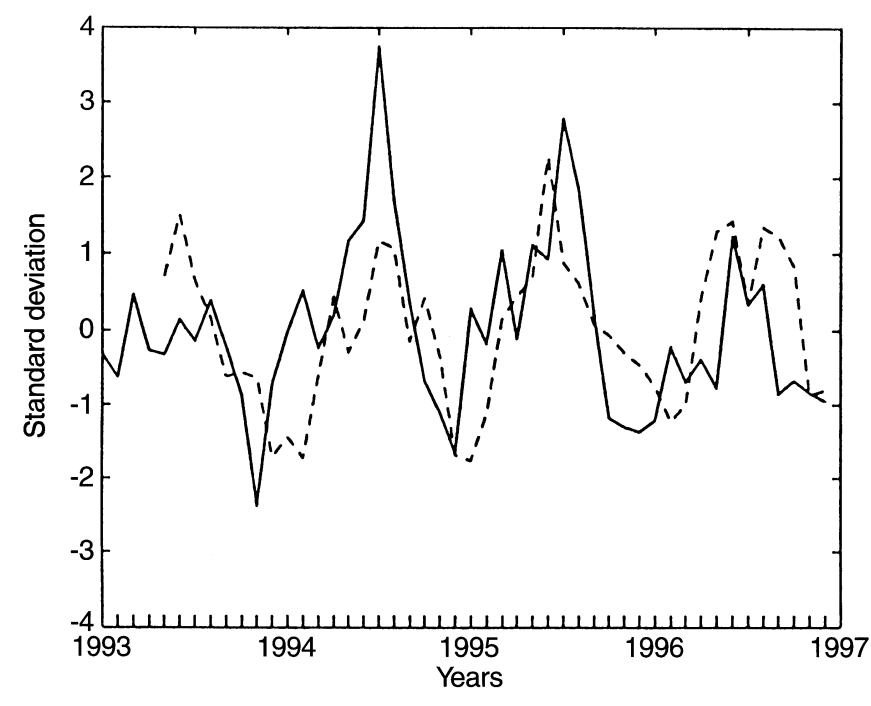

Fig. 4. Normalised differences between day-time and night-time monthly means of the PG (solid line) and average SR amplitudes for the first three modes (dashed line) for the same interval as in Fig. 2

means determined for individual hours (by displaying the curves in Fig. 2 one by one), slight indications of peaks could be detected near to the autumn period especially when regarding curves for certain early morning hours only. In these hours, the PG values are quite low, local thunderstorms hardly occur, as well as vertical and horizontal air motions (contributing to the changes in local condensation nuclei content and in air conductivity) are mostly unimfortant in comparison with the changes of these parameters during day-time. Because of the favourable local conditions mentioned, the morning interval between $0500 \mathrm{UT}$ and $0800 \mathrm{UT}$ has been selected for tracing the appearance of the annual and especially semiannual component in the PG during the four years investigated by using a powerful filtering method. The curve given by solid line in Fig. 5 shows the changes in the averages of monthly mean PG determined for this period. For this curve, the same filtering technique as described by Sátori and Zieger (1996) has been applied for extracting both the annual and the semiannual component. As could be expected, the annual component (indicated by dashed line in Fig. 5) clearly shows the winter maxima and summer minima in PG corresponding to the seasonal variation of local air conductivity (as discussed previously). In the four years investigated, the semiannual component (indicated by dotted line) mostly displays maxima in February and August, i.e. they appear at least one month earlier than those detected regularly in spring and autumn in the case of SR amplitudes. In 1995, the maxima shift even more (to January and July). Thus, the phase of the semiannual signal appearing in the PG differs from that found in the SR amplitudes. It should be mentioned that various pieces of evidence suggested a stronger semiannual signal in rainfall and lightning in Africa, while this signal should be weaker in Asia. From this point of view, the selection of the African diurnal interval seemed to be advisable for investigating the

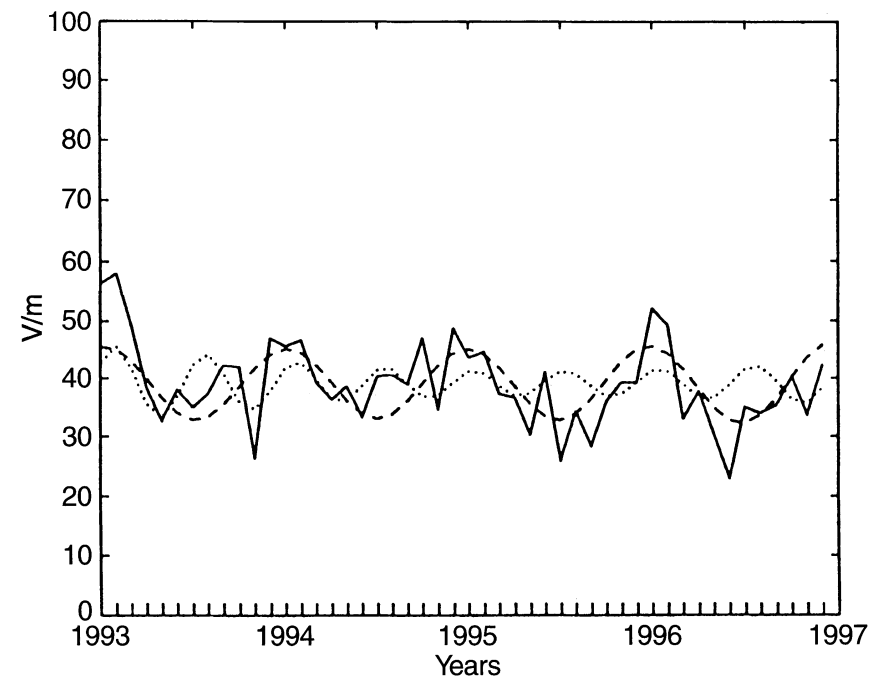

Fig. 5. Monthly means of the PG (in $\mathrm{V} / \mathrm{m}$ ) based on data between 05 UT and 08 UT (solid line), filtered annual component (dashed line) and filtered semiannual component (dotted line) for the same interval as in Fig. 2

semiannual signal in PG. Nevertheless, results (not shown here) derived for this interval did not confirm this supposition. As can be seen in Fig. 5, the use of morning PG values (when a contribution to global thunderstorm activity is from the Asian and Australian continent as e.g. shown by Israël, 1973) led to promising results. This might be due to the fact that during the selected morning interval the local conditions at Nagycenk are more advantageous in terms of finding a semiannual signal in the PG parameter. It is also remarkable that Adlerman and Williams (1996) showing the monthly means of airEarth current for Mauna Loa station detected some type of semiannual signal. The February-March maximum (also in this case) occurred too early to fit the variation pattern of surface air temperature between $\pm 25^{\circ}$ latitude (Williams, 1994), however, the fall maximum appearing in October-November was somewhat similar to the air temperature pattern. Taking into account the results of the present study and those of Adlerman and Williams (1996), it can be concluded that a semiannual signal (corresponding to that found in tropical surface air temperature) is certainly present in SR which is the measure of the AC global circuit, while it is masked or even questionable in DC parameters.

\subsection{Diurnal variation and short-term changes in the $P G$ and in Schumann resonances}

A further comparison between the PG and the SR relative amplitudes is reasonable on the diurnal time scale, too. For this purpose, undisturbed days have been chosen from three different seasons. (It was also required that both the hourly averages of $\mathrm{PG}$ and appropriate SR amplitude data should be continuously available for the chosen days.) Based on reports of a nearby $(10 \mathrm{~km})$ meteorological station of the Hungarian Meteorological Service, clear fair-weather conditions 
were found e.g. for winter on January 14, 1995, for summer on July 25, 1995 and for autumn on October 26, 1995. In all three cases, an anticyclone was dominant over Hungary, the airmass was reported to be clean and mostly cloud-free; these conditions yielded continuous sunshine hours during day-time and no precipitation.

Figure 6 shows the hourly averages of the PG for these days (hours are given in UT). It is evident from the curves that the mean levels are different; this is partly connected with the seasonal variation of the PG. Minima appearing between 0200 and $0600 \mathrm{UT}$ as well as maxima in the interval from 1400 to 1900 UT generally correspond to the main features of the unitary diurnal variation in the atmospheric electric DC global circuit. All curves hint at low-noise measurements on the selected days; (additionally, it should also be considered that hourly values determined on the basis of individual measurements can be compared with an accuracy of about $10 \mathrm{~V} / \mathrm{m}$ because of certain deficiencies common in the reading of analogue records). In spite of the fluctuating values around the minimum, the winter curve especially confirms that the run of the 'Carnegie Curve' is well reproduced by a single day's PG data if local environmental and meteorological conditions are highly favourable. The minima are flat and rather stable both in summer and autumn. The maximum of the summer curve appears somewhat earlier than that of the winter curve and the maximum is broader in the autumn curve. Differences regarding the latter features can be attributed partly to local sources, partly to regional thunderstorm activities which might be accentuated differently in the global activity from time to time.

As regards the curves presented in Fig. 6, it seems that the phase of the diurnal variation of the fairweather PG is quite consistent with that of the 'Carnegie Curve', however, the amplitude of the former is essentially larger than the diurnal amplitude of the latter. This is likely due to local influences. As can be seen e.g. in Fig. 37 of Israël (1957), air conductivity varies with local time showing higher values at night than in daytime hours, i.e. the phase of its diurnal variation is

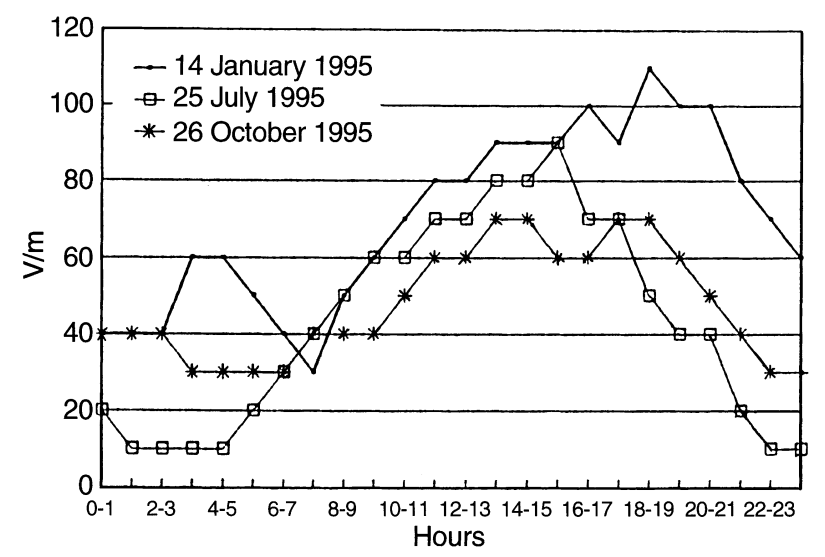

Fig. 6. Diurnal variation of the $P G$ (in $\mathrm{V} / \mathrm{m}$ ) on fair-weather days in three different seasons: winter (January 14, 1995), summer (July 25, 1995) and autumn (October 26, 1995); hours mean UT here and in Figs. 7, 8 opposite to that of the PG. As also known from Israël (1957), sometimes the diurnal amplitude variation of local air conductivity is around $100 \%$ (in percent of mean), i.e. it is rather large as compared to that of the inversely running 'Carnegie Curve'. Thus, it can be assumed that the large amplitude of the diurnal variation of the Nagycenk PG is associated with a similar behaviour of air conductivity (but in opposite sense), even if data for the latter parameter are not available at the corresponding measuring site. It is to be mentioned that the amplitude of the PG diurnal variation is large not only in the case of observations made at the Earth's surface, but also in the case of measurements at higher atmospheric levels. Namely, dual-aircraft measurements carried out by Markson (1985) over ocean and coastline yielded both a potential variation at a height of $1.5 \mathrm{~km}$ and an ionospheric potential $\left(\mathrm{V}_{\mathrm{I}}\right)$ variation which similarly followed in phase the run of the 'Carnegie Curve'. However, the amplitude variation was rather noisy and large at $1.5 \mathrm{~km}$ height over both the ocean and coastline locations, as compared to the variation of the full ionospheric potential at both locations. The range percent variation (range divided by mean) at $1.5 \mathrm{~km}$ height was near to $100 \%$, i.e. twice of that of the comparable full $\mathrm{V}_{\mathrm{I}}$ variation. Considering these results of Markson (1985), the large diurnal amplitude variation shown for the PG measured at the ground (Fig. 6) should not be astonishing, as Nagycenk represents a land station. In PG values determined at land stations, the global signal is generally masked by superposed local effects. This might certainly be influential in respect of the diurnal amplitude variation, too. Nevertheless, measurements carried out with due care together with suitable data selection and treatment could contribute to the preservation of a certain global signal content in the Nagycenk data and to the occasional detection of global features.

In Fig. 7a, b and c, the diurnal variations of the relative amplitudes of the vertical electric component in the first three SR modes are plotted for the same days as for the PG in Fig. 6. Like the PG, the SR amplitudes generally show minima in the interval between 0200 and 0600 UT in each case. Depending on season, the occurrence times of main maxima of the SR amplitudes are somewhat variable, they are less stable than those of the minima; nevertheless, a loose connection with the timing of corresponding PG maxima is discernible. The existing differences can be attributed partly to the effects of local origin in the case of the PG, partly to the changes in source positions affecting the SR parameter.

Excellent fair-weather conditions (like those allowing the demonstration of quite undisturbed diurnal variations in the previous figures) do not occur very often. There are several days on which the PG variation is highly disturbed by strong local influences. Changes in local air conductivity (associated with temperatureforced convection and changing aerosol content), effects due to space charges and electrified clouds, charged precipitation etc. are factors which can mask the global signal content of the PG considerably; as a consequence its normal diurnal variation will essentially be modified, 

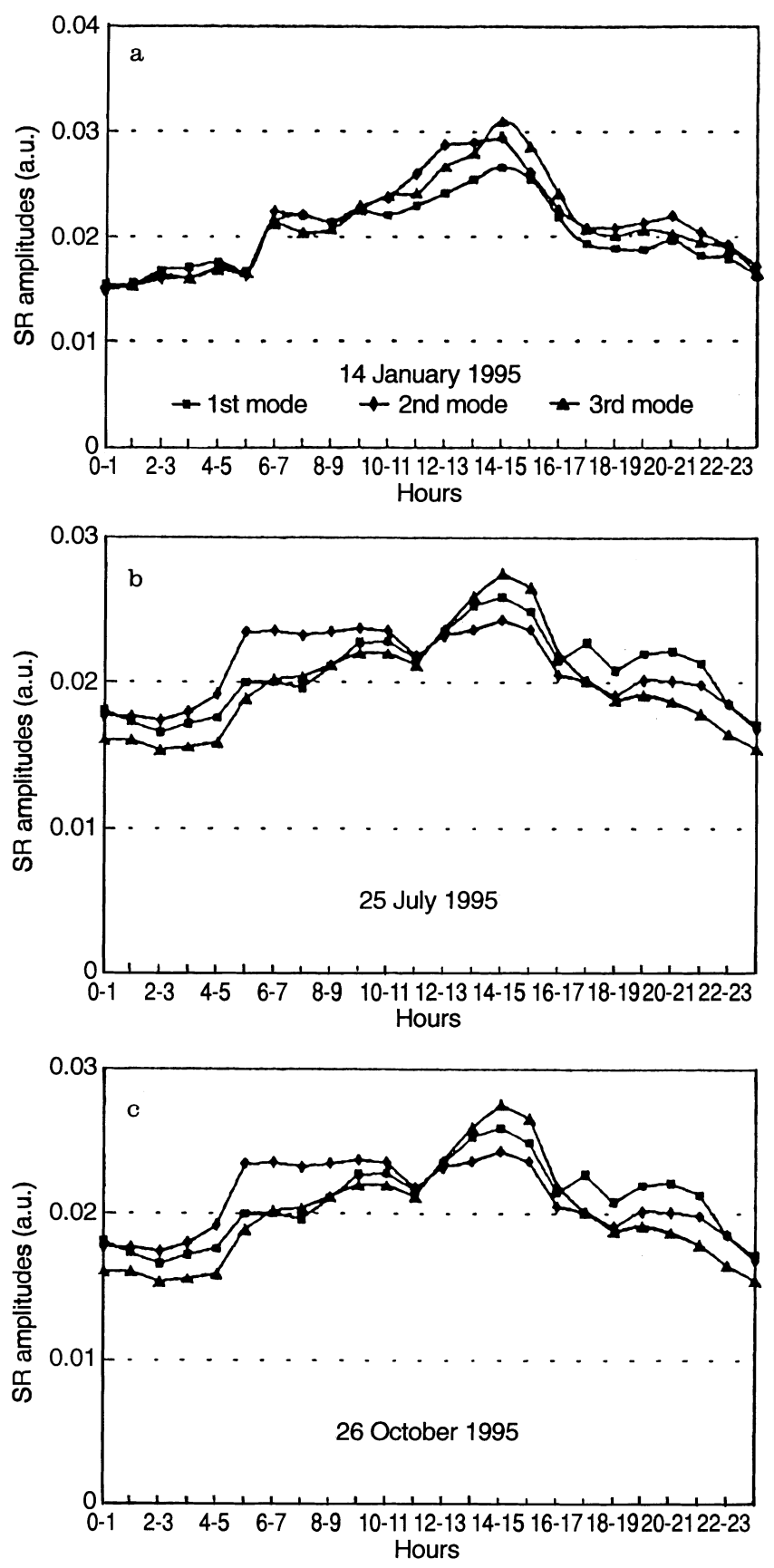

Fig. 7a-c. Diurnal variation of the SR amplitudes (in three modes) on the same days as for the PG in Fig. 6: a winter day, $\mathbf{b}$ summer day, c autumn day

too. Similarly, the diurnal variations of SR amplitudes are not always as regular as those presented previously; they are occasionally contaminated by irregular excursions in quite different parts of the day. On this basis, it seemed worthwhile to look for disturbances simultaneously occurring in the PG and in the SR amplitudes. Several cases of this kind were found, however, only one of them will be presented.

In addition to the hourly averages of the PG, we usually determine the difference between the maximum and the minimum $P G$ values within each hour i.e. the variation range (PGR). This quantity defines the vari- ance well (Unruhe) of the PG and its changes indicate local electrical activity mostly connected with meteorological influences. The measuring range of the PG equipment is limited in such a way that the maximum difference (i.e. PGR-max) can reach about $700 \mathrm{~V} / \mathrm{m}$. In these cases the polarity change of the $P G$ is also considered; large differences are usually associated with strongly electrified clouds or local thunderstorm activity. (Unfortunately, the PG sensor does not allow special detection of the lightning activity, on the basis of the frequency of field changes they produce.)

Defining disturbed days on the basis of enhanced PGR values, the corresponding daily variations of the SR relative amplitudes were generally also disturbed, as was discovered from a thorough inspection of data of the year 1994. For demonstration, a disturbed summer day has been chosen, however, days of enhanced PG activity could be found in each season. Figure 8 shows the daily variations of the SR amplitudes for all three modes on August 20, 1994, together with the variation of the hourly PG range. The strongest enhancement of PGR appears in the hours 0400 and $0500 \mathrm{UT}$; simultaneously the SR amplitudes suddenly increase in all the three modes, however, they are the largest in the first mode. The meteorological conditions were rather disturbed as could be learned from the report of a nearby station of the Hungarian Meteorological Service. The simultaneous enhancements of the PGR and the SR amplitudes in the early morning were connected with a heavy rain shower extending over the measuring area, i.e. the measurements of both parameters were influenced by local meteorological effects. This figure represents a mixed case, namely PGR values return to a low level at $0700 \mathrm{UT}$ and remain there during the whole day, but some further excursions appear in the SR amplitudes (between 1400 and 1700 UT, as well as at 2000,2200 and 2400 UT) hinting at the fact that these disturbances are not directly associated with PG changes, they originate from other sources. Based on a general meteorological report for this day, thunderstorm activity occurring in south-western Hungary (at a

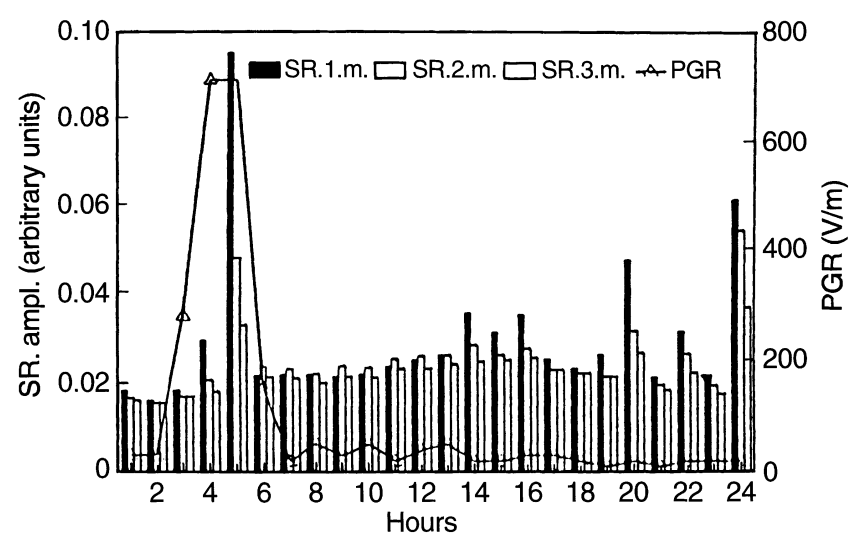

Fig. 8. Hourly relative amplitudes of the SR vertical electric component in the first three modes for a disturbed summer day (August 20, 1994) and hourly PG ranges (PGR, in V/m) for the same day 
distance of about $100 \mathrm{~km}$ from the measuring site) is most likely responsible for the enhanced SR amplitudes.

\section{Discussion and conclusions}

Continuous recording (from May 1993 to December 1996) of the Schumann resonances yielded an appropriate data set for confirming the characteristics previously detected by Sátori and Zieger (1996) in the seasonal variation of the amplitudes of the SR vertical electric component. Based on an initial data set, it was found that the annual variation is apparently present in all three SR modes, and the semiannual variation is significant in the first and second modes, while it is weakly present in the third mode (Sátori and Zieger, 1996). The present study additionally shows the annual variation of the PG observed at the same station. As expected on the basis of earlier results (Märcz and Bencze, 1981), its phase is opposite to that appearing in the SR amplitudes. The annual variation with winter maximum and summer minimum in the PG can be attributed to the opposite seasonal variation of local air conductivity (coupled with a seasonal variation in condensation nuclei being in phase with the PG). The summer maximum in the SR amplitudes is partly a response to the intrinsic global source, (i.e. to the increased global thunderstorm activity in this season) and partly the response to the source proximity effect. As mentioned, Adlerman and Williams (1996) using the entire data set of the Carnegie and Maud measurements have shown that the mean potential gradient in fact peaks during the Northern Hemisphere summer rather than in winter. Moreover, they have shown that the summer maximum in air-Earth current measured at Mauna Loa is also consistent with the annual variation of thunder days for the globe. Results of the present study revealed this summer peak in the Nagycenk PG, too. Indications for the presence of such a signal were rather pronounced in the years 1994 and 1995, and their coincidence with the summer maxima of SR amplitudes is apparent.

Taking also into account the results of Adlerman and Williams (1996), the summer peaks of PG shown in Fig. 3 (based on day-time data) and in Fig. 4 hint at the fact that DC and AC parameters occasionally respond in a similar way to the global source. This seems to be valid in spite of the limited global signal content of the DC parameter as compared with that of the AC component. In Fig. 3, the PG seasonal variation is displayed both by the curve based on night-time data and by that based on day-time data. In accordance with the diurnal variation of the $\mathrm{PG}$, the night-time data show a lower amplitude than the day-time data, however, the winter maxima are apparent in both. Because of the inverse relation between PG and air conductivity, the PG maxima really appear to correspond to local conditions, namely to the presumedly low air conductivity in winter. As air conductivity increases in summer, the PG has to decrease in this season. The clear minima shown by the night data of $\mathrm{PG}$ in consecutive years (Fig. 3, lower curve) are consistent with this expectation. The decreasing tendency is also shown by the day-time data (Fig. 3, upper curve) hinting at an association with the behaviour of local air conductivity, however, the pronounced PG peaks emerging from the trend in summer can be attributed to global thunderstorm activity rather than to local influences. Namely, in the data selection procedure, data affected by local thunderstorm activity and some other strong meteorological disturbances were properly excluded.

From the seasonal variation of early morning PG data, it might be suspected that secondary maxima appearing near to autumn would indicate the presence of a weak semiannual signal (shown by the solid line curve in Fig. 5). Applying a suitable filtering technique, both an annual and a semiannual signal could be extracted from this curve. The maxima in the semiannual component occurred too early to fit the variation pattern of the semiannual component in SR amplitudes (showing maxima around spring and autumn). For comparison it is worth noting that Williams (1994) has found positive correlations between parameters of the atmospheric electric global circuit and surface air temperature (for the tropics and for the globe) on both semiannual and annual time scales. Averaging monthly mean surface temperatures (on $5^{\circ} \times 5^{\circ}$ latitude - longitude squares, considering land and ocean alike) over the latitude interval $\pm 25^{\circ}$, he showed a seasonal variation for area-averaged dry-bulb temperature with two distinct maxima in April and October. A similar curve was presented for the seasonal variation of wet-bulb temperature. Averaging over the $\pm 60^{\circ}$ latitude interval with the same data set, an estimate of the global seasonal variation in air temperature was obtained. The results showed a distinct annual variation with a maximum in Northern Hemisphere summer. Williams (1994) argued that lightning is the global circuit quantity which is most responsive to temperature. Using the monthly mean number of thunder days (for stations within $\pm 25^{\circ}$ latitude and $\pm 60^{\circ}$ latitude), he detected a semiannual signal with maxima in April and October, in phase with the temperature maxima; additionally, an annual signal with maximum in Northern Hemisphere summer was also present for $\pm 60^{\circ}$ latitude. Midnight lightning data and global lightning flash-rate based on SR measurements also showed a good agreement with temperature variations, however, other parameters connected with the DC global circuit (ionospheric potential and airEarth current) correlated less well with temperature variations on semiannual and annual time scales. The semiannual signal in ionospheric potential shown by Williams (1994) is rather weak and the autumn bump is more distinct than the spring one. (It is to be mentioned that the ionospheric potential measurements were few in number and the variance in each monthly mean was substantial.) This partly resembles the seasonal run of unfiltered morning PG data (Fig. 5, solid line). Williams (1994) using the air-Earth current data of Hogg (1950) showed the semiannual variation in this DC parameter, too. It is admitted that air-Earth current is more 
globally representative than the electric field; notwithstanding in the semiannual variation of this parameter the autumn peak is also more pronounced than the spring one. According to Williams (1994), the seasonal amplitude of DC circuit parameters was smaller than that of lightning-related parameters. Results of the present study seem to confirm that in tracking the semiannual and annual variations of global air temperature, the SR amplitudes (representing lightning-related, AC component) show better agreement than the PG (manifesting the DC component).

Further results of Williams (1992) convincingly indicated for several stations in the tropics that lightning activity increases nonlinearly with surface air temperature. Moreover, evidence has been provided that SR (being a lightning-related phenomenon and related to the global electric circuit) can be applied as a sensitive measure of tropical air temperature fluctuations. Plotting simultaneous values of the ionospheric potential $\left(\mathrm{V}_{\mathrm{I}}\right)$ and SR, Williams (1992) showed a strong association between these two parameters that supported the idea that both include a global component. Later, Price (1993) used two independent data sets of satellite surface temperature measurements as a substitute for true surface air temperatures and showed that they correlate well with variations in global thunderstorm activity. Considering the previous results of Williams (1992), as well as presuming linear relation between global lightning frequencies and ionospheric potential, Price (1993) suggested that a $1 \%$ increase in global surface temperature would result in a $20 \%$ increase in ionospheric potential. Results of Markson and Price (1996) confirmed the positive correlation between ionospheric potential and global surface temperature obtained from three different data sets. They also showed that ionospheric potential is positively correlated with an inferred global lightning/deep cloud index which correlates with global temperature. Based on these and previously mentioned results, certain parameters connected with the atmospheric electric (AC and DC) global circuit (like SR and $\mathrm{V}_{\mathrm{I}}$ ) can be regarded as proper tools in tracking the global temperature fluctuations. The present study analysing SR and PG variations yielded some results which seem to confirm that SR may be responsive to temperature variations, while simultaneous measurements of atmospheric electric AC and DC parameters can be useful for detecting peculiarities in both components which are comparable under advantageous conditions.

Comparing diurnal variations of the Nagycenk PG (on selected fair-weather days of different seasons, in Fig. 6) with those of relative amplitudes in the first three SR modes (Fig. 7), certain similarities can also be found on this time scale. Thus, it is apparent that minima simultaneously appeared in both parameters. Maxima of the individual PG curves occurred nearly in phase with the maximum of the unitary diurnal variation in the atmospheric electric DC global circuit (Fig. 6). It should be mentioned that the diurnal variation of the PG for the selected winter day particularly confirms that the run (phase) of the 'Carnegie Curve' is repeatable by a single day's PG data. For such favourable cases, it may be supposed that the PG data are less spoiled by local environmental and strong meteorological influences. When regarding the phase of the PG diurnal variation it can be concluded that the global signal content of PG is quite well preserved, however, this is questionable when comparing the amplitude variation of the PG with that of the 'Carnegie Curve' because of reasons discussed in Sect. 3.2. Nevertheless, careful selection and treatment of the PG data can lead to acceptable results regarding PG variations on longer (e.g. annual and semiannual) time scales.

A detailed analysis of one year's data both for the PG and the SR vertical electric component detected a number of simultaneous short-term changes in the two parameters; sometimes the changes appeared only in one of them, however. The individual changes generally lasted a few hours and they occurred in different parts of the day. It may be presumed that changes in air pollution certainly influence the PG (as local air conductivity is affected) but the SR parameter will not be influenced in such cases; while effects associated with strong winds can hamper both measurements. Strong meteorological disturbances, like local thunderstorms, heavy rain or snow showers will certainly perturb both parameters. More distant thunderstorm activity should not affect the PG measurements, however, it can generate changes in the SR parameter. For one example (given for August 20, 1994) in Fig. 8, it could be stated (on the basis of the corresponding meteorological report) that localized meteorological disturbances were really responsible for the short-term changes simultaneously appearing in parameters of the DC and AC global circuit. In the early morning of the actual day, changes appeared in both parameters due to the effect of a local heavy rain shower; while in the afternoon and evening hours, the SR amplitudes alone showed an extraordinary behaviour which could be attributed to lightning occurring at a distance of about $100 \mathrm{~km}$ from the observation site (as also learned from the corresponding meteorological report). Consequently, the SR amplitudes seem to be more responsive to perturbing sources outside the immediate vicinity of the observation site than the case for the PG parameter. Changes also appearing in the latter, however, can help to identify those sources which are confined to the measuring area. Actually, the analysis of the Nagycenk data hints at the fact that the comparison of the simultaneous SR and PG records should enable us to reject intervals with local influences. Thus, this kind of disturbed data can be excluded from SR studies aimed at detection of global responses, e.g. at the determination of further relations between changes in SR parameters and surface air temperature as previously reported by Williams (1992).

Atmospheric electric parameters derived from ground-based measurements made at land stations are certainly affected by local influences that are investigated in the present study. Notwithstanding, continuous service of the measuring equipment, standard calibration and data processing, as well as careful data 
selection can yield a data base suitable for investigating also global circuit signatures from a land station. This seems to be confirmed by certain results of the present study.

Acknowledgements. The investigations were supported by the Hungarian State Grant, T 4395, and by the Hungarian Space Agency under the Space Weather Programme. The authors wish to thank the referees for their valuable advice in preparing the present version of the study.

Topical Editor L. Eymard thanks S. Chauzy and E. A. Williams for their help in evaluating this paper.

\section{References}

Adlerman, E. A., and E. R. Williams, Seasonal variation of the global electrical circuit, J. Geophys. Res., 101, 29679-29688, 1996.

Ádám, A., and P. Bencze, Investigation of a low-frequency natural electromagnetic energy source, (in Russian), Acta Techn. Hung., 43, 357-365, 1963.

Bencze, P., and F. Märcz, Atmosphärisch-elektrische und ionosphärische Messungen im Observatorium bei Nagycenk, Observatoriumsberichte des Geophysikalischen Forschungslaboratoriums der Ungarischen Akademie der Wissenschaften vom Jahre 1966, Sopron, 143-152, 1967.

Bencze, P., and F. Märcz, The Geophysical Observatory near Nagycenk, II. Atmospheric electric and ionospheric measurements, Acta Geod. Geophys. Mont. Hung., 16, 353-357, 1981.

Chalmers, J. A., Atmospheric Electricity, Pergamon Press, London, Paris, New York, 1957.

Dolezalek, H., The World Data Centre for Atmospheric Electricity and Global Change Monitoring, Selection of data for collecting or application (or the "fairweather hour syndrome"), Appendix III, Draft, Preprint, London, 1-13, 1991.

Hogg, A. R., Air-earth current observations in various localities, Arch. Meteorol, 3, 40-55, 1950.
Israël, H., Atmosphärische Elektizität, I. Grundlagen, Leitfähigkeit, Ionen, Akademische Verlagsgesellschaft, Geest and Portig K.G., Leipzig, 1957.

Israël, H., Atmospheric Electricity, II. Fields, Charges, Currents, Israel Program for Scientific Translations, Jerusalem, 1973.

Koenigsfeld, L., and N. Piraux, Un nouvel electrometre portaif pour la measure des charges electrostatiques par systeme electronique, Inst. R. Meteorol. Belg. Mem. XLV, 1951.

Markson, R., Aircraft measurements of the atmospheric electrical global circuit during the period 1971-1984, J. Geophys. Res., 90, 5967-5977, 1985.

Markson, R., and C. Price, Ionospheric potential as a proxy index for global temperature, In Proceedings of the 10th International Conference on Atmospheric Electricity, Osaka, Japan, ICAE and SAEJ, 632-635, 1996.

Märcz, F., and P. Bencze, Variations of the atmospheric electric potential gradient at the Nagycenk observatory, Acta Geod. Geophys. Mont. Hung., 16, 415-422, 1981.

Price, C., Global surface temperatures and the atmospheric electrical circuit, Geophys. Res. Lett., 20, 1363-1366, 1993.

Sátori, G., Monitoring Schumann Resonances, II. Daily and seasonal frequency variations, J. Atmos. Terr. Phys., 58, 14831488, 1996.

Sátori, G., and B. Zieger, Spectral characteristics of Schumann resonances observed in Central Europe, J. Geophys. Res. 101, 29663-29669, 1996.

Sátori, G., J. Szendröi, and J. Verö, Monitoring Schumann Resonances, I. Methodology, J. Atmos. Terr. Phys., 58, 14751481, 1996.

Wait, J. R., Mode theory and propagation of v.l.f. radio waves. Nat. Bur. Stand. 64 D, (4) 387-404, 1960.

Williams, E. R., The Schumann resonance: a global tropical thermometer, Science, 256, 1184-1187, 1992.

Williams, E. R., Global circuit response to seasonal variations in global surface air temperature, Mon. Weather Rev., 122, 19171929, 1994.

Williams, E. R. and N. Renno, Conditional instability, tropical lightning, ionospheric potential, and global change, In 19th Conference on Hurricanes and Tropical Meteorology, Miami, American Meteorological Society, Boston, Mass., 36-42, 1991. 